\title{
Cachexia-Anorexia Syndrome and Food Supplementation in Cancer Patients
} \author{
Lovasic ${ }^{4}$ \\ ${ }^{1}$ Department of Radiotherapy and Oncology, Clinical Hospital Center Rijeka, Croatia \\ ${ }^{2}$ Department of Gastroenterology, Clinical Hospital Center Rijeka, Croatia \\ ${ }^{3}$ Departement Centre Medico-Social Pointe Noire, Congo \\ ${ }^{4}$ Department of Radiotherapy and Oncology Clinic, Croatia
}

Renata Dobrila-Dintinjana $^{1 *}$, Marin Golčićc ${ }^{1}$, Aleksandar Čubranić ${ }^{2}$ and Marijan Dintinjana ${ }^{3}$, Ingrid Belac

Submission: January 13, 2017; Published: February 03, 2017

*Corresponding author: Renata Dobrila-Dintinjana, Professor of Medicine, Department of radiotherapy and oncology, Clinical Hospital Center Rijeka, Krešimirova, Croatia, Tel: +385-98-257-729; Email: renatadobrila@windowslive.com

\begin{abstract}
Loss of appetite and weight are one of the first symptoms of a malignant disease. In rapidly advancing cancer which does not respond to therapy, severe cachexia will undoubtedly lead to death. The cancer tissue and the body itself secrete cytokines and other factors, which, combined interfere with both central and peripheral metabolic pathways causing anorexia and cachexia. Assessing nutritional state in cancer patients should be made before starting oncological treatment, but also regularly during the course of therapy. The treatment of cachexia and anorexia in cancer patients is a complex issue and includes specific treatment with a goal of reducing cancer volume, providing nutritive support and intervening with pharmaceuticals. There are different appetite-enhancing medications with the most effective being megestrol acetate, corticosteroids and cannabinoids. Eicosapentaenoic acid (EPA) on the other hand is the key in treating cachexia.
\end{abstract}

Today, formulations with EPA are prepared in such ways that they can be mixed with other food and can be prepared by following many different recipes. Parenteral nutrition is also an option, but is rarely used due to its high cost and risk of infections, and should not be used in end-stage disease. Administering nutritive advice, prescribing nutritional and pharmacological support can temporarily stop weight loss and improve appetite, quality of life and social interactions of cancer patients, and allow for a longer administration of systemic anti-tumor therapy and hence probably for a longer survival.

Keywords: Anorexia; Cachexia; Cancer; Eicosapentaenoic acid; Megestrol acetate; Nutrition; Quality of Life

Abbreviations: BMI: Body Mass Index; CACS: Cancer Anorexia-Cachexia Syndrome; CS: Cortico Steroids; CRP: C-Reactive Protein; DPA: Docosahexaenoic Acid; EPA: Eicosapentaenoic Acid; IFN-y: Interferon Gamma; IL: Interleukine; kCal Kilocalorie; LMF: Lipid-Mobilising Factor; MA: Megestrol Acetate; NSTT: Nottingham Screen Tool Test; NF-kB: Nuclear Factor kappa B; PIF: Proteolysis Inducing Factor; PN: Parenteral Nutrition; QoL: Quality of Life; TNF-alpha: Tumor Necrosis Factor alpha; ZAG: Zinc Alpha-Glycoprotein

\section{Introduction}

Loss of appetite and weight are one of the first symptoms of a malignant disease. Malnutrition is a common finding in cancer patients and its incidence (8-88\%) depends on the site of the primary cancer. Head and neck along with gastrointestinal cancer patients tend to suffer mostly from malnutrition, which is responsible for death of $20-40 \%$ of these patients [1]. Cancer Anorexia-Cachexia Syndrome (CACS) is defined as a multi-factorial syndrome combining anorexia and cachexia symptoms, featuring loss of appetite with progressive loss of body weight on account of skeletal muscular mass loss, functional decay of tissue, along with inability to gain weight even with an increased intake of food [2]. Cachexia is not a 'steady state' and does not happen overnight. It begins with loss of appetite (anorexia) crossing into pre-cachexia with early clinical and/or metabolic signs of cachexia, with mild loss of body weight, which, unless an intervention is made, progresses into severe cachexia.

The term cachexia signifies an involuntary loss of more than $5 \%$ of body weight in last 6 months or a combination of involuntary loss of more than $2 \%$ of body weight along with decreased body mass index (BMI). In rapidly advancing cancer which does not respond to therapy, severe cachexia will undoubtedly lead to death [3]. Cancer patients can develop different mechanical abnormalities in the digestive tract (obstruction, stenosis, gastroparesis etc.) which, in addition to pain and nausea, prevent intake and utilization of 
nutrients. However, even in patients with no digestive tract abnormalities, specific biochemical and metabolic changes due to cancer lead to weight loss and declining of nutritional status even in early phase of malignancy. Presence of malnutrition in cancer patients is a risk factor when performing different diagnostic or therapeutic measures and increases toxicity of systemic chemotherapy and radiotherapy. The loss of fat and muscle mass leads to progressive functional weakness.

\section{Discussion}

Cancer tissue secretes cytokines such as interleukine-6 (IL-6) and tumor necrosis factor alpha (TNF-alpha), circulating hormones, neuropeptides, neurotransmitters and other factors that lead to increased catabolism and anorexia. Along with secretion from tumor tissue, as a response to presence of a cancer, the body itself secretes pro-inflammatory cytokines which influence central metabolic pathway directed from hypothalamus and peripheral metabolic pathways, directly influencing lipolysis and proteolysis. Due to an elevated level of cytokines, hypothalamus is unable to respond to peripheral signals which result in over-activation of metabolic pathways supporting anorexia, while pathways supporting an increased appetite are inhibited [4].

Even in the microenvironment of the cancer, different abnormalities supporting both local and systemic inflammation are identified. In tumor cachexia, an increase of C-reactive protein (CRP) levels above $10 \mathrm{mg} / \mathrm{L}$ is an indirect, but significant sign of both systemic inflammation and poor prognosis. In tumor microenvironment there is an increase of secretion of pro-inflammatory cytokines such as TNF-alpha and pro-catabolic cytokines such as zinc alpha-glycoprotein (ZAG) which are, along with host-response factors such as interferon gamma (IFN-y), responsible for degradation of skeletal muscle and fatty tissue.

Intact metabolism of lipids is very important for cancer, due to a necessity to form stabile membranes of proliferating cells and utilize different signal pathways. Lipid-mobilising factor (LMF) is of paramount importance for cancer proliferation and survival. Combination of LMF and ZAG factors leads to an increase of lipid mobilisation and activation of oxidative pathways in mitochondria of brown adipose tissue, causing lipolysis, waste of energy and hypercatabolism [5,6]. On the other hand, proteolysis inducing factor (PIF), working through ubiquitin proteasome pathway, is responsible for degradation of muscle proteins and myosin depletion (along with unchanged levels of actin protein). Additionally, an increase of skeletal muscle degradation is also achieved exploiting the mechanism of insulin resistance [7]. An assessment of nutritive status in cancer patients is a basis for determining pre-cachexia state and prevention of further development to cachexia. Assessing nutritional state should be made as early as possible, at least before starting chemotherapy or radiotherapy, but also regularly during the course of the disease.
Although there are numerous more or less detailed questionnaires and methods for determining nutritional status, for clinical practice, the most appropriate methods need to be simple and quick, such as Fearon questionnaire (if patient unintentionally lost more than $5 \%$ of body weight in 3 to 6 months, if food intake was lower than $1500 \mathrm{kCal}$ per day, and if CRP values are above $10 \mathrm{mg} / \mathrm{L}$, tumor cachexia is present) or Nottingham Screening Tool Test (NSTT). Using these methods we can determine if a patient is in nutritive risk or is on a verge of developing cachexia. Along with before-mentioned factors, if a patient is suffering from a severe inflammation such as sepsis or pneumonia, or is required to undergo an operation, the risk for developing cachexia increases significantly [8].

The treatment of CACS is a complex issue and includes specific treatment with a goal of reducing cancer volume, providing nutritive support and intervening with pharmacological options. In those patients with a high risk for developing CACS, an introduction of special nutritional formulations is required, while patients in pre-cachexia state require additional pharmacological therapy [9]. In both of those groups, regular nutritional screening should be undertaken, while nutritive support and pharmacological treatment should be administered at least until a clinical improvement is noted or until there are no more risk factors (e.g. finalization of chemotherapy).

There are controversies regarding the utilization of nutritional support in end-stage disease, where it might not be appropriate to administer appetite-enhancing medications, while basic nutritive support should be applied up to the level appropriate to each patient individually. There are different appetite-enhancing medications with the most effective being megestrol acetate (MA), a semi-synthetic progesterone which, by enhancing the appetite, indirectly causes an increase in body weight. An exact mechanism of MA action is still not elucidated, but it is generally thought that MA stimulates appetite by reducing the secretion of pro-inflammatory cytokines (IL1, IL-6 and TNF-a) and by enhancing the stimulation of neuropeptides in hypothalamus [10]. Although corticosteroids (CS) are efficient in improving appetite and general wellbeing, its long term use is limited by the numerous long-term side-effects such as insulin resistance and development of iatrogenic diabetes mellitus, fluid retention, steroid myopathy, skin fragility, and adrenal insufficiency and cognitive defects.

Hence, the use of CS is recommended only for a short period of time, especially as the effect on appetite last around 4 weeks. Cannabinoids such as dronabinol are also effective in reducing anorexia however, around $15 \%$ of patients are unable to resume with the therapy due to various side effects [11]. Research is currently undergoing on many different medications which effect appetite and weight loss, such as bortezomib (NF-kB transcription factor inhibitor), grelin (a natural ligand for human growth factor receptor), melanocortin antagonist (inhibition of TNF-alpha), anti IL- 6 antibodies, thalidomide, 
anabolic steroids (oxandrolon) and amorelin (human growth hormone agonist), which proved to be very effective in several studies. Appetite-stimulating medications most likely function by interacting through endorphin receptors and interfering with IL-1 stimulation, along with activation of cannabinoid receptors which are enclosed in neurochemical circle of prostaglandin synthesis inhibition and leptin regulation.

If administered at the right time, nutritive advice, food supplementation and pharmacological support can stop weight loss, improve the appetite, life quality and potentially the outcome of the disease $[12,13]$. Eicosapentaenoic acid (EPA) has a key role in treating pre-cachexia and cachexia as proven by many clinical studies [14]. EPA, omega-3-fatty acid and docosahexaenoic acid (DHA) improve appetite and body weight (on account of dry body mass) and lead to an increase in level of physical activity and general quality of life (QoL). The effects are not limited to administration of MA, as both EPA and DHA have immunomodulatory effects and suppress production of pro-inflammatory cytokines, and while DHA modulates steroid receptor line in human lines of cancer cells, EPA inhibits the consequential effects of LMF and PIF. Omega-3-fatty acids show multiple anti-tumor effects in vivo on different stages of cancer development such as cellular proliferation, cellular survival, angiogenesis, inflammation, metastasizing and epigenetic abnormalities, all which are necessary steps for progression of malignant disease. The daily dose of EPA required to stop the process of cachexia and for the patient to start gaining weight is around $1.5-2.2$ grams per day.

Along with body weight gain, a reduction of inflammatory parameters is reported by different clinical studies [15]. There are many different formulations with EPA on the market. The most researched one for now is Pro Sure $\mathrm{R}$ nutritive formulation, with over 20 clinical studies reporting positive effects in stopping cachexia process and gaining body weight, along with an improvement in QoL and reduction of levels of inflammatory parameters. In general, patients gained around $1-2 \mathrm{~kg}$ of body weight. This leads to an improvement of patients' physical strength and better tolerance of oncological treatments, with lesser number of side-effects. Social momentum most not be forgotten as well, as food intake, along with being a necessity for survival, is an important social manifestation. Cancer patients with an improved appetite due to MA and with a better physical condition due to nutritive support participate more often in social interactions and have an improved life satisfaction [16].

Today, formulations with EPA are prepared in a such ways that they can be mixed with other food (chocolate, fruit, juices etc.), and can be prepared by following many different recipes, all with goal to satisfy organoleptic needs of a patient and to reduce the possibility of developing aversion towards 'artificial foods'. Formulations with EPA are not alone sufficient considering necessary calorie intake; their administration must be combined with other enteral formulations rich with calories, which combined together, will satisfy caloric needs of a cancer patients. Enteral formulations are specially designed for different patient populations, so some contain more fibres (for patients suffering from constipation), while others have reduced level of carbohyd rates (for diabetic patients) etc. When the digestive tract of a cancer patient is functioning, enteral support should be administered through mouth (per oral) or via nasogastric tube or percutaneous stoma (usually for patients with advanced head and neck patients or oesophageal and gastric patients).

Parenteral nutrition (PN) is used when a cancer patient suffers from severe emesis, severe abdominal pain, profuse diarrhea, and mechanical obstruction in digestive tract or other bowel pathology (e.g. fistula); in these conditions enteral nutrition is contraindicated. However, PN is rarely used in cancer patients due to high cost, increased sensitivity to systemic infection and high morbidity up to $15 \%$, although its use in postoperative setting should be reconsidered due to the effect of EPA on reduction of inflammation and inflammatory complications. In terminal phase of advanced malignancy, PN should not be used, while enteral nutrition should be used as long as possible. In the end stage, PN could potentially result in minimal advances in prolonging the survival; however without an increase of QoL and any long term solutions, this would subject patients to dysthanasia [17].

\section{Conclusion}

Administering nutritive advice, prescribing nutritional and pharmacological support can temporarily stop weight loss and improve appetite, quality of life and social interactions of cancer patients, and allow for a longer administration of systemic anti-tumor therapy and hence probably for a longer survival. Nutritive support alleviates some of the problems of cancer patients and their families have, raise QoL and allows for better social interactions.

\section{References}

1. Von Haehling S, Anker SD (2010) Cachexia as a major underestimated and unmet medical need: facts and numbers. J Cachexia Sarcopenia Muscle 1(1): 1-5.

2. Tuca A, Jimenez-Fonseca P, Gascón P (2013) Clinical evaluation and optimal management of cancer cachexia. Crit Rev Oncol Hematol 88(3): 625-636

3. Fearon K, Strasser F, Anker SD, Bosaeus I, Bruera E et al. (2011) Definition and classification of cancer cachexia: an international consensus. Lancet Oncol 12(5): 489-495.

4. Wu Q, Clark MS, Palmiter RD (2012) Deciphering a neuronal circuit that mediates appetite. Nature 483(7391): 594-597.

5. Tisdale MJ (2009) Zinc-alpha2-glycoprotein in cachexia and obesity. Curr Opin Support Palliat Care 3(4): 288-293.

6. SA Beck, MJ Tisdale (1991) Lipid mobilising factors specifically associated with cancer cachexia. Br J Cancer 63(6): 846-850.

7. Cabal-Manzano R, Bhargava P, Torres-Duarte A, Marshall J, Bhargava P, et al. (2001) Proteolysis-inducing factor is expressed in tumours of 
patients with gastrointestinal cancers and correlates with weight loss. Br J Cancer 84(12): 1599-1601.

8. Blum D, Strasser F (2011) Cachexia assessment tools. Curr Opin Support Palliat Care 5(4): 350-355.

9. Del Fabbro E (2010) More is better: a multimodality approach to cancer cachexia. Oncologist 15(2): 119-121.

10. Ruiz Garcia V, López-Briz E, Carbonell Sanchis R, Gonzalvez Perales JL, Bort-Marti S (2013) Megestrol acetate for treatment of anorexiacachexia syndrome. Cochrane Database Syst Rev 3: CD004310.

11. Fearon KC (2008) Cancer cachexia: developing multimodal therapy for a multidimensional problem. Eur J Cancer 44(8): 1124-1132.

12. Davis M, Lasheen W, Walsh D, Mahmoud F, Bicanovsky L, et al. (2012) A Phase II dose titration study of thalidomide for cancer-associated anorexia. J Pain Symptom Manage 43(1): 78-86.

13. Madeddu C, Mantovani G, Gramignano G, Macciò A (2015) Advances in pharmacologic strategies for cancer cachexia. Expert Opin Pharmacother 16(14): 2163-2177.
14. Krznarić I, Juretić A, Šamija M, Dintinjana-Dobrila R, Vrdoljak E, et al. (2007) Croatian guidelines for use of eicosapentaenoic acid and megestrol acetate in cancer cachexia syndrome. Lijec Vjesn 129(12): 381-386.

15. Barber MD, Ross JA, Voss AC, Tisdale MJ, Fearon KC (1999) The effect of an oral nutritional supplement enriched with fish oil on weight-loss in patients with pancreatic cancer. Br J Cancer 81(1): 80-86.

16. Murphy RA, Yeung E, Mazurak VC, Mourtzakis M (2011) Influence of eicosapentaenoic acid supplementation on lean body mass in cancer cachexia. Br J Cancer 105(10): 1469-1473.

17. Muscaritoli M, Molfino A, Laviano A, Rasio D, Rossi Fanelli F (2012) Parenteral nutrition in advanced cancer patients. Crit Rev Oncol Hematol 84(1): 26-36.

\section{Your next submission with Juniper Publishers will reach you the below assets}

- Quality Editorial service

- Swift Peer Review

- Reprints availability

- E-prints Service

- Manuscript Podcast for convenient understanding

- Global attainment for your research

- Manuscript accessibility in different formats

( Pdf, E-pub, Full Text, Audio)

- Unceasing customer service

Track the below URL for one-step submission https://juniperpublishers.com/online-submission.php 